\title{
FACTORES ESTRESORES EN ESTUDIANTES DEL PREGRADO DE LA FACULTAD DE CIENCIAS CONTABLES - UNMSM
}

\author{
STRESSOR FACTORS IN UNDERGRADUATE STUDENTS OF THE \\ ACCOUNTING SCIENCES FACULTY - UNMSM \\ Ana María Gutiérrez HubY* \\ Docente Principal de la Facultad de Ciencias Contables \\ Universidad Nacional Mayor de San Marcos - UNMSM / Lima - Perú \\ [Recepción: Agosto 2016/ Conformidad: Setiembre 2016]
}

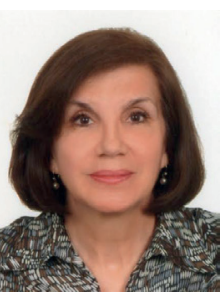

\section{RESUMEN}

El objetivo del presente artículo expone la problemática del estrés académico en los estudiantes del Pregrado de la Facultad de Ciencias Contables de la UNMSM. Se basa en los primeros resultados obtenidos del estudio "Estrés académico, determinación y estrategias de solución para el área económico empresarial para la universidad de San marcos y el Centro Universitario de los Lagos", que desarrolla la autora en el presente año, en el cual se abordan los posibles estresores académicos causantes del estrés. En el método se empleó como muestra a 353 estudiantes del primer y noveno semestre de estudios, de los que, a través de un análisis factorial de componentes, se determinó información objetiva sobre los niveles de estrés presentes en la población estudiada. Teniendo como resultados que la mitad de la población encuestada evidencia estrés, agudizándose en un $2.8 \%$ de los estudiantes. Se determinaron nueve factores estresores, de los cuales "la imposibilidad de participar en las decisiones respecto del propio trabajo académico", "una falta de control sobre el propio rendimiento académico" y la "sobrecarga académica de los estudiantes", fueron identificadas como los más recurrentes causantes del estrés. Asimismo, concentran mayores niveles de estrés los estudiantes del noveno semestre, y las mujeres, en cuanto a distinción según género.

\section{Palabras Clave:}

Estrés académico, Estudiantes pregrado, Factores estresores.

\begin{abstract}
This article exposes the problem of academic stress in undergraduate students of the Faculty of Accounting Sciences of the UNMSM. It is based on the first results obtained from the study "Academic stress, determination and solution strategies for the Business Economic Area for the University of San Marcos and the University Center de los Lagos", developed by the author this year, where the possible academic stressors are addressed. The study sample consisted of 353 students from the first and ninth semester of studies. Through a factorial analysis of components, the objective information on the stress levels present in the studied population was determined. The results show that half of the population surveyed present stress, aggravated in $2.8 \%$ of the students. Nine stress factors such as "the inability to participate in decisions about their own academic work", "a lack of control over their own academic performance" and "students' academic overload ", were identified as the most recurrent stressors. Likewise, students in the ninth semester concentrate higher levels of stress, and women, in terms of gender distinction.
\end{abstract}

\section{Keywords:}

Academic stress, Undergraduate Students, Stressor factors.

\footnotetext{
* Doctora en Administración. Universidad de San Pedro. Magíster en Administración con Mención en Gestión Empresarial - UNMSM. E-mail: agutierrezh@unmsm.edu.pe Colaboradora: Iris Aguilar Alonso. Bachiller en Estadística - UNMSM Colaboradora: Elsa Violeta Rafael Díaz. Técnico en Computación
} 


\section{INTRODUCCIÓN}

\section{La sociedad, fuente constante de estrés}

Según nos refiere Cabanyes (2012) ${ }^{1}$, el estrés está instalado y se manifiesta de manera creciente en esta actual sociedad globalizada, la cual experimenta un incremento de trastornos de ansiedad, depresión e insomnio cada vez mayores, ocasionados en parte por la incorporación de nuevas demandas, muchas de ellas irreales generadas por agentes externos de la industria, la cual hace a los miembros de esta sociedad estar más atados a una vida consumista que desequilibra su situación financiera complicando su existencia, haciéndola encontrar o percibir como insuficientes los recursos disponibles. Por otra parte, estos trastornos de ansiedad también son ocasionados por la velocidad en que la que se vienen produciendo los cambios en todos los órdenes, tecnológicos, políticos, educativos, etc., haciendo que la vida y el trabajo estén cada vez más ligados y condicionados por el factor tiempo, trayendo ello cambios negativos en las condiciones de vida y de trabajo de sus habitantes.

En este contexto, son acontecimientos del día a día, la inseguridad en las calles, el trabajo prolongado debido a una fuerte presión laboral, ya sea por el temor a la pérdida del empleo o estatus laboral, el endeudamiento con las tarjetas de crédito por los bajos ingresos, la poca solvencia económica para mantener a la familia, el tener que atender diversas cosas al mismo tiempo, la falta de habilidades, la exigencia de una cada vez más rápida capacidad de adaptación y de respuesta a los problemas,tienen como común denominador, ser causantes de una continua tensión y malestar, es decir, estrés, el cual al acumularse en muchos casos es detonante de cuadros depresivos en las personas que incluso pueden llegar hasta el suicidio.

En el ámbito empresarial, el estrés se presenta con sus propias implicancias, sobre el recurso humano, primando en las competencias laborales, que según Gamero (2010, p.20) vienen cambiando incesante y constantemente, pues cada vez se establecen nuevas prácticas de labor orientadas a mejorar la productividad y la calidad, siendo frecuente que estas nuevas condiciones de productividad y competitividad impuestas por la economía globalizada generen incremento de la presión laboral haciendo progresiva la inseguridad en las condiciones de empleo de los trabajadores.

No obstante, este aspecto, por parte de la mayoría de las empresas, suele no recibir la atención debida por parte de la gerencia directiva, que suelen pasar por alto el estado de estrés que experimentan sus colaboradores, no reparando en los riesgos de esta enfermedad y ello es debido a que el estrés no ha estado siendo un problema muy perceptible, habiéndosele subestimado en sus efectos negativos para la empresa y para la economía.

Hoy en día, la situación ha mejorado, gracias a importantes estudios y a estadísticas respecto al tema, muchos de los cuales se enfocan en los altos costos de pérdidas que significa para la empresa, por falla en la calidad y cantidad de los productos y servicios, ausentismos de trabajadores, cambios de cargos, deserciones laborales, accidentes de trabajo, jubilaciones prematuras o incluso el deceso de los trabajadores. Además del aumento en los gastos en servicios de salud por envejecimiento prematuro, enfermedades, uso indebido de drogas, tramitación de divorcios, violencia y conflictos laborales, entre otros.

Otros estudios, por su parte, destacan los altos costos que representa el estrés para las empresas,en términos de pérdida de oportunidades para el aprovechamiento del talento humano,señalan además que los empleados sometidos al estrés, son menos receptivos, siendo su angustia un freno para la creatividad, optando por mantener una postura de ser seguidores en lugar de ser líderes y tomar riesgos, siendo una de sus conclusiones señalar que el estrés inhabilita a las personas para resolver conflictos adecuadamente, haciendo ver que las consecuencias del estrés global pueden empeorar en los próximos años, si no se toman las medidas para menguar la situación.

$\mathrm{Al}$ respecto, los investigadores de esta temática también han aportado con alternativas de solución. En este sentido, las organizaciones pueden hacer mucho para ayudar a sus empleados en su bienestar tanto físico como mental, disminuyendo el estrés entre sus integrantes y disminuyendo los costos que 
este representa. $\mathrm{Al}$ respecto la Organización Internacional del Trabajo, se ha pronunciado, señalando que: "las empresas que ayuden a sus empleados a hacer frente al estrés y reorganicen con cuidado el ambiente laboral en todos sus ámbitos, tienen más posibilidades de lograr ventajas competitivas.

Por lo referido hasta este punto, el estrés ha trascendido a casi todos los ámbitos de la vida, el ámbito social, económico, laboral, empresarial, político; no obstante en el presente artículo se le aborda desde el ámbito académico, en el cual se ha instalado con sus propias particularidades y repercusiones.

\section{El estrés en el ámbito académico}

El estrés en el ámbito académico, en especial en el nivel universitario, suscita particular interés por su afectación en la salud mental de los miembros de la comunidad académica, considerándose aquí a estudiantes, docentes y personal administrativo. Asimismo, también en el contexto académico la cultura organizacional de la institución educativa, es materia de análisis.

En el caso de los estudiantes, particularmente, por considerárseles los profesionales del futuro, éstos son objeto de estudio para la temática del estrés, habiéndose desarrollado en torno a ellos numerosos estudios y desde diversos enfoques, sobre todo aquellos que lo analizan desde sus características psicológicas, que por lo general, señalan su vulnerabilidad al desarrollo de ciertas patologías (Reynaldos, 2006; Guavita, 2006; Kamali etál., 2002, citados por Martín G., M. \& Trujillo A., F., 2012, p. 1518).

En el caso de los docentes, la importancia del estudio del estrés es visto porque la afectación en la salud de estos profesionales trasciende en su rendimiento laboral, pues un docente estresado se encontrará menos motivado para desempeñar sus funciones adecuadamente, siendo sensible este aspecto, ya que en sus manos descansa la responsabilidad de la formación de los nuevos profesionales, de tal forma que su bienestar mental es una variable fundamental para el crecimiento en materia de cali- dad educativa(Herranz-B., J., Reig, F., A., Cabrero, G., J., 2006, p. 746) ${ }^{2}$.

Los estudios desarrollados en torno a estos actores de la formación profesional, reportan como principales situaciones estresantes para los docentes, la excesiva carga de trabajo y la falta de tiempo, además de otros factores como materiales de trabajo inadecuados o insuficientes, las exigencias ergonómicas, las restricciones en torno a la toma de decisiones que pueda hacer la institución académica, sobre la forma en la que desarrollan sus propias tareas docentes, así como el excesivo alumnado a su cargo, las dificultades de aprendizaje de los alumnos, su indisciplina, además de las presiones sociales y personales, entre otros.

En el caso del personal administrativo, por ser un miembro activo de la organización educativa, este personal, debe ir a la par del desarrollo dinámico y productivo actual, siendo considerado el talento humano que contribuye al desenvolvimiento de los procesos administrativos, porque es la imagen institucional al estar en contacto con los usuarios internos y externos, por ende las exigencias y la alta presión son parte de la cotidianidad de los trabajadores administrativos de las instituciones universitarias, haciéndose éstas presiones excesivas y difíciles de controlar.

En el caso de la cultura organizacional, se advierte que toda organización tiene una cultura implícita que define a sus miembros las normas de conducta que deben asumir, por tanto, la cultura organizacional como sistema de valores y metas, contribuye también a generar condiciones favorables o desfavorables de trabajo, las cuales pueden condicionar estrés en el entorno.

Para el caso del presente artículo, son los estudiantes el centro de atención para el estudio del estrés, el cual, según lo refiere Barraza ${ }^{3}, 2007$ (citado por Díaz M., Y. (2010), el estrés puede ser concebido en general como un fenómeno de tipo adaptativo, dado que el modo de afronte que puedan tener los estudiantes sobre aquellas situaciones que alteran su estabilidad emocional, en su estancia académica, determinará su grado de adaptación a los factores

2 Departamento de Psicología de la Salud. Universidad de Alicante.

3 Licenciado en educación de personas con problemas de aprendizaje y Maestro en Educación con campo en la Práctica Educativa. Universidad Pedagógica de Durango. 
estresantes. Cuando el estudiante no tiene esta capacidad de afrontar o neutralizar las situaciones estresantes, por no contar con los recursos necesarios para hacerlo, entonces es cuando surge en él la situación de estrés, de ahí que de las actitudes que los universitarios de hoy adopten ante la vida nacerán las que tendrán en un futuro.

Por lo tanto, aquellos alumnos que logren asimilar bien los cambios que conlleva el ámbito universitario aprenderán de la situación y se enriquecerán personalmente tomando dichos cambios como un desafío, con un nivel controlado de estrés, que les ayudará a llevar a cabo sus acciones; pero, por el contario, aquéllos que no puedan manejar correctamente estas situaciones, experimentarán insatisfacción personal y desgaste, es decir, el estrés académico.

En este sentido, el presente artículo que constituye parte de los resultados del estudio 2016 denominado "Estrés académico, determinación y estrategias de solución para el Área económico empresarial de la universidad de San marcos y el Centro Universitario de los Lagos", a cargo de esta autora, desarrolla la afectación que el entorno educativo universitario, con sus presiones, demandas y disfunciones puede llegar a influir sobre la salud, el bienestar y el rendimiento académico de los estudiantes del área contable, considerando la progresiva alta exigencia académica que distinguen a la carrera, debido en parte al afán de su involucramiento en procesos de cumplimiento de estándares para acreditación de calidad, lo cual requiere del alumnado importantes esfuerzos de adaptación para una mejor preparación académica, fundamental para el futuro profesional, en un mercado laboral cada vez más competitivo.

De esta manera, se exponen los resultados del estudio que ha abarcado dos grupos vulnerables de estudiantes, los que inician el proceso de estudios y los que están por concluir su proceso formativo, desenvolviéndose ambos grupos en un período de transición y cambio que son la adolescencia y adultez primaria, siendo otro de los propósitos contar con bases objetivasque permitan más adelante, perfilar la necesidad de intervenir con programas preventivos de atención a este síndrome como evidencia del inte- rés e intención lógica de ayudar a los estudiantes de esta Carrera del área empresarial, tal como lo señalan la mayoría de los estudiosos de esta temática quienes demandan de las instituciones una mayor atención en el cuidado de la salud mental de sus estudiantes.

En la actualidad las universidades se encuentran inmersas en procesos de evaluación y acreditación los cuales valoran y promueven la calidad del servicio educativo, siendo el estudio del estrés relevante por cuanto se relaciona a la calidad de vida de los miembros de la comunidad universitaria, lo que redunda en el aporte que se pretende dar al concientizar y aportar a la calidad educativa de una universidad pública como San Marcos.

\section{FUNDAMENTACIÓN TEÓRICA}

Maslach y Jakcson, 1981 (citados por Rodríguez G. M., et al $)^{4}$, autores que presentaron una definición sólida del síndrome burnout, aludiendo a un estado de estrés agudo y crónico,refieren acerca de este estrés, el estar constituido por tres dimensiones: el cansancio o agotamiento emocional, referido a una reducción de recursos emocionales, acompañado de manifestaciones somáticas y psicológicas, como la depresión, la ansiedad y la irritabilidad; la despersonalización, que se refiere al desarrollo de actitudes negativas, y, finalmente, la falta o disminución de la realización personal, en referencia a la percepción de que las posibilidades de logros han desaparecido.

A este respecto, Selye, 1950; Fidalgo, 2006; Hernández, 2009 (citados por Rodríguez G., M., San Miguel S., M., Muñoz M., A., Rodríguez R., C. (2014), exponen la conclusión de que el síndrome del estrés, denota vinculación directa entre estímulos negativos que alteran significativamente la configuración del organismo y sus respuestas, fisiológicas y conductuales.

En términos generales, el estrés académico puede afectar a cualquier nivel educativo, no obstante a la hora de afirmar que un estudiante universitario padece estrés o referir que no lo padece debe sustentarse en base a un conocimiento rigurosamente profundo. (Polo, Hernández y Pozo, 1995), es por ello que en relación al estudio del estrés académico, en especial

4 Médica internista , Doctora en Ciencias de la Educación, , docente investigadora de la Facultad de Medicina de la Universidad Autónoma de Coahuila, Unidad Torreón. 
cuando se centra en los estudiantes, éste no puede considerarse únicamente desde una única perspectiva, sino que debe ser considerado en conjunto con los aspectos biológicos, psicológicos y educacionales. Fue Lazarus ${ }^{5}$, 1966, 1991b; Lazarus y Folkman, 1984 (citados por Rodríguez G., M., San Miguel S., M., Muñoz M., A., Rodríguez R., C. (2014), quienes establecieron que no es el factor estresor el causante de estrés, sino más bien la percepción que el individuo posee sobre el estresor. Es entonces la evaluación cognoscitiva que hace la persona sobre un estímulo estresor lo que define la situación, tanto para la reacción o respuesta estresante como para el afrontamiento posterior.

Basados en el modelo general de estrés psicosocial de Lazarus y Folkman, se puede afirmar que se trata pues de un complejo fenómeno que implica la consideración de variables interrelacionadas: estresores académicos, experiencia subjetiva de estrés, moderadores del estrés académico y finalmente, efectos del estrés académico.

En el ámbito académico se ha encontrado que el apoyo social podría actuar, además, como un potenciador en el logro de un desempeño satisfactorio de los estudiantes (Román y Hernández, 2005, citado por Felman, L. et al (2008), ya que contribuiría a que afronten los estresores propios de su ambiente con mayores probabilidades de éxito (Labrador, 1995, en Martín, 2007).

Específicamente, los estudiantes de Medicina sufren un importante estrés desde el inicio de su carrera, y si bien es aceptable cierto grado de tensión, no todos los estudiantes lo resuelven de manera adecuada (Ferrer et al. 2002, citado por Rodríguez G., M. et al (2014). En muchos de ellos, los programas y las exigencias generan miedo, incompetencia, enojo y sensación de inutilidad y culpa, todas manifestaciones que pueden producir respuestas psicológicas y físicas mórbidas.

Frente a esto, los estudiantes emplean diversos mecanismos para superar y procesar el estrés; algunos de ellos producen consecuencias negativas, como la evasión del problema, la idealización de las situaciones (interpretación de los acontecimientos como a la persona le gustaría que fueran y no como realmente son), el aislamiento social y la autocrítica excesiva. Por el contrario, las estrategias que incluyen afrontar el problema, la comunicación y el respaldo por parte de terceros y la manifestación de las emociones, constituyen todos enfoques positivos que reducirán el estrés. (Dyrbyeet al., 2005, citado por Rodríguez G., M. et al (2014).

En cuanto a los estresores académicos se entiende que son todos aquellos factores o estímulos del ambiente organizacional educativo (eventos, demandas, etc.) que presionan o sobrecargan de algún modo al estudiante. En concreto, el presente estudio se centra en el medio universitario, indagando sobre las posibles fuentes de estrés en aspectos tales como los exámenes y evaluaciones, el rendimiento académico, las relaciones sociales, la sobrecarga de trabajo, metodología docente o la falta de control sobre el propio entorno educativo.

Podemos decir así, que el sujeto evalúa diversos aspectos de su entorno educativo como amenazas o retos, como demandas a las que puede responder adecuadamente o para las que cree no tener los recursos necesarios, como factores que están o no bajo su control, etc. Sin embargo, no todos los estudiantes universitarios padecen estrés. La respuesta al estrés depende de la disposición personal para hacer frente a las demandas de la situación así como de la propia situación a la que nos refiramos. Es decir, cómo un individuo responde a los acontecimientos estresantes depende tanto de la disposición personal de dicho individuo como de la situación en la cual dichos acontecimientos suceden.

\section{METODOLOGÍA}

\section{Población y Características}

La muestra de la población estudiada, la conformaron 353 estudiantes del Pregrado del Primer y Noveno Semestre de estudios de las Escuelas Académico-Profesionales de Contabilidad, Gestión Tributaria y Auditoría Empresarial y del Sector Público de la Facultad de Ciencias Contables de la Universidad Nacional Mayor de San Marcos. Su distribución, por

5 Psicólogo influyente en la historia del campo y profesor emérito de Psicología de la Universidad de California, Berkeley. Falleció el 24 Noviembre en California, a la edad de 80 años. 
Escuelas y Semestres de estudios, se presenta de la siguiente manera: A La EAP de Contabilidad, pertenecen 154 estudiantes, que constituyen el $43.7 \%$ de la totalidad de la muestra de la población estudiada.

De esta muestra, 103 estudiantes, que representan el 66.9\% de la muestra para esta Carrera, cursan el Primer Semestre y 51, es decir 33.1\%, cursan el Noveno Semestre. La muestra total para la EAP de Gestión Tributaria, lo constituyen 83 estudiantes que representan el 23.5\%; de esta muestra, 50 (60.2\%), cursan el Primer Semestre y 33 (39.8\%), cursan el Noveno Semestre. Por último, la muestra para la EAP de Auditoría Empresarial y del Sector Público lo constituyen 116 estudiantes que representan el $32.86 \%$ de la totalidad de la muestra estudiada, de ellos, 61 (52.5\%), pertenecen al Primer Semestre y $55(47.4 \%)$ al Noveno Semestre de estudios (ver Tabla 01).

Tabla No 01

Distribución de estudiantes por semestre, según EAP

\begin{tabular}{|l|c|c|c|}
\hline \multirow{2}{*}{ EAP } & \multicolumn{2}{|c|}{ Semestre } & \multirow{2}{*}{ Total } \\
\cline { 2 - 3 } & Primero & Noveno & \\
\hline \multirow{2}{*}{ Contabilidad } & 103 & 51 & 154 \\
& $66,9 \%$ & $33,1 \%$ & $100,0 \%$ \\
\hline \multirow{2}{*}{ Gestión Tributaria } & 50 & 33 & 83 \\
& $60,2 \%$ & $39,8 \%$ & $100,0 \%$ \\
\hline Auditoría Empresarial y & 61 & 55 & 116 \\
del Sector Público & $52,6 \%$ & $47,4 \%$ & $100,0 \%$ \\
\hline \multirow{2}{*}{ Total } & 214 & 139 & 353 \\
& $60,6 \%$ & $39,4 \%$ & $100,0 \%$ \\
\hline
\end{tabular}

Fuente: Resultados de Encuesta. Elaboración propia.

Como características de esta población encuestada, los resultados de la sección de datos generales del cuestionario aplicado, exponen que el $46 \%$ de toda la población estudiada, son varones y el $54 \%$ son mujeres. La edad promedio de los estudiantes del Primer Semestre es de 19 años y la de los estudiantes del Noveno Semestre es de 23 años. En cuanto al estado civil, el $99 \%$ son solteros y sólo el 1\% convivientes y padres. En cuanto a su procedencia, el $84 \%$ procede de la Costa, el 13\%, de la Sierra y el 3\%, de la Selva. Asimismo, la gran mayoría (79\%) viven con sus padres aún, el $6 \%$ señaló vivir sólo con hermanos, el $10 \%$ con otros familiares y el $5 \%$ viven solos. En cuanto a información brindada en el aspecto laboral, en el caso de los estudiantes del Primer Semestre, el $13 \%$ trabaja un promedio de 5 horas, mientras que en el caso de los estudiantes del Noveno Semestre, el 75\% está trabajando, haciéndolo un promedio de 7 horas. Respecto a su desenvolvimiento académico, el 19\% de estudiantes de los estudiantes del Primer Semestre, manifestó que en su primer examen habían experimentado la desaprobación de al menos alguna asignatura, en el caso de los estudiantes del Noveno Semestre refirieron que en el transcurso de su carrera al menos habían desaprobado alguna asignatura.

\section{Instrumento}

El cuestionario que se aplicó en la recolección de los datos, fue diseñado por Cabanach, R.G., Valle A., Piñero, I., Rodríguez,S. y García, $M^{6}$. (2009) y fue administrado de forma anónima. El cuestionario está estructurado en tres secciones diferenciadas. La primera sección está compuesta de 11 preguntas sobre datos generales de los estudiantes; la segunda sección, lo conforman 53 enunciados sobre situaciones o acontecimientos que en el ámbito académico, podrían producir estrés en los estudiantes encuestados, lo cuales pueden ser valorados como amenaza para su bienestar emocional; la tercera y última sección, la constituyen 22 enunciados que describen estados de ánimo en las últimas semanas por parte de los estudiantes, previa a la aplicación de la encuesta. Las 
respuestas planteadas para cada una de las preguntas se miden sobre una escala de 5 puntos, donde 1 , significa "Nunca" y 5 , "siempre".

\section{Técnicas de Análisis de Datos:}

Para determinar los estresores académicos causantes del estrés en la población estudiantil materia de estudio, se sometieron los datos recogidos a través del cuestionario,a la técnica estadística del análisis factorial aplicándose el método de análisis de componentes principales y el método de rotación Varimax con Kaiser, para encontrar grupos homogéneos de variables y obtener una máxima reducción de los factores comunes que representen a la variables ori- ginales recogidas del instrumento aplicado.

De las comunidades obtenidas se pudo comprobar que el modelo factorial está bien representado ya que el $85 \%$ de los ítems o componentes, concentró coeficientes de correlación superiores a 0.5 , lo que indica que cada ítem explica más del $50 \%$ de su variabilidad original en el factor asignado; por otra parte, también se evidenció que sólo el 15\% de ítems explican menos del 50\% de su variabilidad en el factor, lo que supone su reasignación a algún otro factor que mejor explique el modelo, siendo estos ítems, el $5,10,16,26,27,35,46$ y 48 , tal como se aprecia en la Tabla $\mathrm{N}^{\circ} 02$.

Tabla No 02

Comunidades

\begin{tabular}{|c|c|c|}
\hline Ítem & Inicial & Extracción \\
\hline P1 & 1 & 0.71 \\
\hline P2 & 1 & 0.72 \\
\hline P3 & 1 & 0.69 \\
\hline P4 & 1 & 0.66 \\
\hline P5 & 1 & 0.44 \\
\hline P6 & 1 & 0.76 \\
\hline P7 & 1 & 0.72 \\
\hline P8 & 1 & 0.66 \\
\hline P9 & 1 & 0.72 \\
\hline P10 & 1 & 0.48 \\
\hline P11 & 1 & 0.61 \\
\hline P12 & 1 & 0.65 \\
\hline P13 & 1 & 0.68 \\
\hline P14 & 1 & 0.55 \\
\hline P15 & 1 & 0.62 \\
\hline P16 & 1 & 0.26 \\
\hline P17 & 1 & 0.52 \\
\hline P18 & 1 & 0.66 \\
\hline
\end{tabular}

\begin{tabular}{|c|c|c|}
\hline Ítem & Inicial & Extracción \\
\hline P19 & 1 & 0.65 \\
\hline P20 & 1 & 0.68 \\
\hline P21 & 1 & 0.67 \\
\hline P22 & 1 & 0.71 \\
\hline P23 & 1 & 0.61 \\
\hline P24 & 1 & 0.53 \\
\hline P25 & 1 & 0.59 \\
\hline P26 & 1 & 0.49 \\
\hline P27 & 1 & 0.23 \\
\hline P28 & 1 & 0.52 \\
\hline P29 & 1 & 0.60 \\
\hline P30 & 1 & 0.63 \\
\hline P31 & 1 & 0.90 \\
\hline P32 & 1 & 0.50 \\
\hline P33 & 1 & 0.55 \\
\hline P34 & 1 & 0.66 \\
\hline P35 & 1 & 0.27 \\
\hline P36 & 1 & 0.62 \\
\hline
\end{tabular}

\begin{tabular}{|c|c|c|}
\hline Ítem & Inicial & Extracción \\
\hline P37 & 1 & 0.64 \\
\hline P38 & 1 & 0.60 \\
\hline P39 & 1 & 0.71 \\
\hline P40 & 1 & 0.63 \\
\hline P41 & 1 & 0.66 \\
\hline P42 & 1 & 0.73 \\
\hline P43 & 1 & 0.70 \\
\hline P44 & 1 & 0.63 \\
\hline P45 & 1 & 0.65 \\
\hline P46 & 1 & 0.41 \\
\hline P47 & 1 & 0.52 \\
\hline P48 & 1 & 0.46 \\
\hline P49 & 1 & 0.51 \\
\hline P50 & 1 & 0.89 \\
\hline P51 & 1 & 0.59 \\
\hline P52 & 1 & 0.51 \\
\hline P53 & 1 & 0.55 \\
\hline & & \\
\hline & &
\end{tabular}

Fuente: Resultados de Encuesta. Elaboración propia.

\section{Determinación de los Factores Estresores}

Para la determinación del número de factores del análisis, se optó por la distribución de dimensiones con autovalores superiores a 1.2, detectándose así 9 factores que explican el $60.16 \%$ de la varianza total.

El primer factor representa el $10.99 \%$ de la varianza total, el segundo factor representa el 8.34\% de la varianza total (Ver tabla $\mathrm{N}^{\circ} 03$ ), el tercer factor representa el $7.65 \%$ de la varianza total el cuarto factor representa el $7.49 \%$ de la varianza total, el quinto factor representa el $7.02 \%$ de la varianza total, el sexto factor representa el 5.40\% de la varianza total, el séptimo factor representa el 5.14\% de la varianza total, el octavo factor representa el $4.51 \%$ de la varianza total y el noveno factor representa el 3.63\% de la varianza total.

Luego para asignar los ítems a cada factor se consideraron saturaciones mayores a [0.40], donde se encontraron solo 6 ítems que representan a 2 factores que son los ítems 21, 28, 42, 43, 44 y 45 y los demás 
Tabla No 03

Varianza Total explicada

\begin{tabular}{|c|c|c|c|c|c|c|c|c|c|}
\hline \multirow[t]{2}{*}{ Componente } & \multicolumn{3}{|c|}{ Autovalores iniciales } & \multicolumn{3}{|c|}{$\begin{array}{l}\text { Sumas de las saturaciones al cua- } \\
\text { drado de la extracción }\end{array}$} & \multicolumn{3}{|c|}{$\begin{array}{c}\text { Suma de las saturaciones al cua- } \\
\text { drado de la rotación }\end{array}$} \\
\hline & Total & $\begin{array}{c}\% \text { de la } \\
\text { varianza }\end{array}$ & $\begin{array}{c}\% \\
\text { acumulado }\end{array}$ & Total & $\begin{array}{c}\% \text { de la } \\
\text { varianza }\end{array}$ & $\begin{array}{c}\% \\
\text { acumulado }\end{array}$ & Total & $\begin{array}{c}\% \text { de la } \\
\text { varianza }\end{array}$ & $\begin{array}{c}\% \\
\text { acumulado }\end{array}$ \\
\hline 1 & 16.985 & 32.048 & 32.048 & 16.985 & 32.048 & 32.048 & 5.827 & 10.994 & 10.994 \\
\hline 2 & 3.069 & 5.791 & 37.839 & 3.069 & 5.791 & 37.839 & 4.401 & 8.304 & 19.298 \\
\hline 3 & 2.637 & 4.975 & 42.814 & 2.637 & 4.975 & 42.814 & 4.055 & 7.651 & 26.949 \\
\hline 4 & 1.869 & 3.527 & 46.341 & 1.869 & 3.527 & 46.341 & 3.970 & 7.490 & 34.439 \\
\hline 5 & 1.839 & 3.469 & 49.811 & 1.839 & 3.469 & 49.811 & 3.721 & 7.021 & 41.460 \\
\hline 6 & 1.523 & 2.873 & 52.684 & 1.523 & 2.873 & 52.684 & 2.862 & 5.400 & 46.860 \\
\hline 7 & 1.473 & 2.779 & 55.463 & 1.473 & 2.779 & 55.463 & 2.725 & 5.141 & 52.001 \\
\hline 8 & 1.282 & 2.418 & 57.881 & 1.282 & 2.418 & 57.881 & 2.391 & 4.512 & 56.513 \\
\hline 9 & 1.202 & 2.268 & 60.149 & 1.202 & 2.268 & 60.149 & 1.927 & 3.636 & 60.149 \\
\hline 10 & 1.136 & 2.144 & 62.293 & & & & & & \\
\hline 11 & 1.074 & 2.027 & 64.320 & & & & & & \\
\hline 12 & .966 & 1.823 & 66.143 & & & & & & \\
\hline 13 & .924 & 1.743 & 67.886 & & & & & & \\
\hline 14 & .896 & 1.690 & 69.576 & & & & & & \\
\hline 15 & .822 & 1.550 & 71.127 & & & & & & \\
\hline 16 & .804 & 1.517 & 72.644 & & & & & & \\
\hline 17 & .769 & 1.451 & 74.095 & & & & & & \\
\hline 18 & .730 & 1.378 & 75.473 & & & & & & \\
\hline 19 & .700 & 1.321 & 76.794 & & & & & & \\
\hline 20 & .679 & 1.281 & 78.075 & & & & & & \\
\hline 21 & .635 & 1.199 & 79.274 & & & & & & \\
\hline 22 & .603 & 1.138 & 80.412 & & & & & & \\
\hline 23 & .585 & 1.105 & 81.517 & & & & & & \\
\hline 24 & .574 & 1.082 & 82.599 & & & & & & \\
\hline 25 & .564 & 1.065 & 83.664 & & & & & & \\
\hline 26 & .536 & 1.012 & 84.676 & & & & & & \\
\hline 27 & .522 & .984 & 85.661 & & & & & & \\
\hline 28 & .489 & .923 & 86.584 & & & & & & \\
\hline 29 & .484 & .913 & 87.497 & & & & & & \\
\hline 30 & .453 & .855 & 88.352 & & & & & & \\
\hline 31 & .413 & .780 & 89.131 & & & & & & \\
\hline 32 & .410 & .774 & 89.906 & & & & & & \\
\hline
\end{tabular}




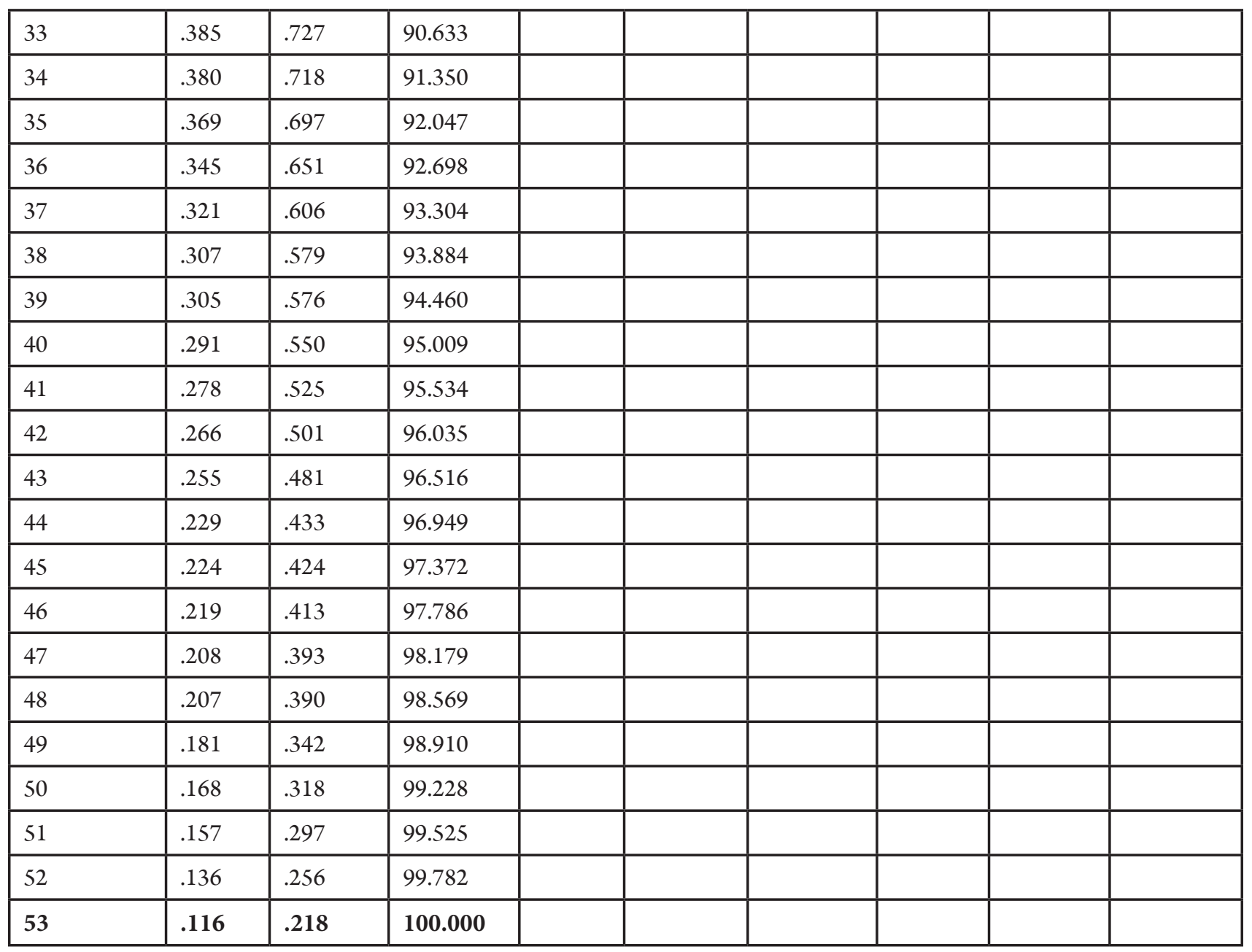

Fuente: Resultados de Encuesta. Elaboración propia. 
ítems representan a un solo factor, lo que indica que el modelo es bien representado con 9 factores. A los ítems que representan a 2 factores se decidió su asignación al factor que guarde mejor relación con los ítems detectados (Tabla $\mathrm{N}^{\circ} 03$ ).

El primer factor está conformado por 9 ítems, denominándose "Baja estima académica" porque sus ítems describen la autoestima de los estudiantes frente a ciertos indicadores que reflejan inseguridad en su ritmo de aprendizaje, obteniendo respuestas como: porque rindo claramente por debajo de mis conocimientos, porque no creo que pueda hacer frente a las exigencias de la carrera que estudio, porque no creo que pueda lograr mis objetivos propuestos, porque desconozco si mi progreso académico es adecuado, porque no sé cómo hacer bien las cosas, porque no sé qué hacer para que se reconozca mi esfuerzo y mi trabajo, porque no tengo claro cómo conseguir que se valore mi dominio de las materias, porque no sé qué hacer para que se reconozca mi valía personal.

El segundo factor "Inseguridad al intervenir en público" estuvo conformado por 5 ítems que describen características del estudiante como: nerviosismo e inquietud cuando le preguntan en clase, cuando tiene que hablar en voz alta en clase, al salir a la pizarra, al hacer una exposición o hablar en público durante un determinado tiempo y el exponer en público alguna opinión.

El tercer factor estuvo conformado por 9 ítems, al que se le denominó "Deficiencia metodológica del profesor" porque describe el nerviosismo que provocan los docentes a los estudiantes por la carencia de metodologías al momento de desarrollar sus clases, siendo algunas respuestas: Cuando el profesor da la clase de una manera determinada y luego nos examina de un modo poco coherente con lo enseñado, cuando los profesores no se ponen de acuerdo entre ellos, cuando no me queda claro cómo he de estudiar una materia, cuando no tengo claro qué exigen en las distintas materias, cuando los profesores plantean trabajos, actividades o tareas que no tienen mucho que ver entre sí, cuando el profesor no plantea de forma clara qué es lo que tenemos que hacer, cuando el profesor plantea trabajos, actividades o tareas que son contradictorias entre sí, cuando los distintos profesores esperan de nosotros cosas diferentes y cuando existe una clara falta de coherencia entre los contenidos de las distintas materias.

El cuarto factor fue denominado: "Sobrecarga académica” estuvo conformado por 9 ítems que resaltan la percepción de la excesiva carga académica que reciben los estudiantes, siendo algunas respuestas: Por el excesivo número de asignaturas que integran el plan de estudios, por las demandas excesivas y variadas que nos hacen, por el escaso tiempo que dispongo para estudiar las distintas materias, por la excesiva cantidad de información que se me proporciona en clase sin que se indique claramente lo fundamental, por el excesivo tiempo que debo dedicarle a la realización de las actividades académicas, porque no dispongo de tiempo para dedicarme a las materias todo lo necesario, por la excesiva carga de trabajo que debo atender, por el excesivo número de horas de clase que tengo a diario y por el ritmo de trabajo o estudio que se nos exige.

El quinto factor tiene 6 ítems, al cual se le denominó "malas relaciones sociales en el contexto académico”. Este ítem describe el desfavorable ambiente social que perciben los estudiantes, dando como respuestas: porque no tengo posibilidad alguna o es muy escaso el dar mi opinión sobre la metodología de enseñanza de las materias del plan de estudios, por los conflictos en las relaciones con otras personas, por la excesiva competitividad existente en clase, por la falta de apoyo de los compañeros, por la ausencia de un buen ambiente en clase y por la existencia de favoritismos en clase.

El sexto factor "temor a los exámenes" tiene 4 ítems que vincula las malas relaciones con los exámenes, obteniéndose respuestas como: cuando tengo exámenes, mientras resuelvo los exámenes y cuando se acercan las fechas de exámenes.

El séptimo factor "Imposibilidad de participar en las decisiones respecto del propio trabajo académico" compuesto por 4 ítems. Las respuestas fueron: cuando el profesor espera de nosotros que sepamos cosas que no nos ha enseñado, cuando el profesor da por hecho que tenemos conocimientos que en realidad no tenemos, cuando el profesor plantea exámenes claramente incongruentes con lo estudiado/enseñado y porque las posibilidades de opinar sobre el 
procedimiento de evaluación de las asignaturas del plan de estudios son muy escasas o nulas.

El octavo factor "carencia de valor de los contenidos de aprendizaje y estudio" conformado por 4 ítems, que agrupan respuestas como: que las asignaturas que cursamos tienen escaso interés, que lo que estoy estudiando tiene una escasa utilidad futura, que las clases a las que asisto son poco prácticas y porque no está en mis manos plantear los trabajos, tareas o actividades como me gustaría.
Y un noveno factor "Falta de control sobre el propio rendimiento académico" compuesto por 3 ítems, que agrupa respuestas como: porque los resultados obtenidos en los exámenes no reflejan, en absoluto, mi trabajo anterior de preparación ni el esfuerzo desarrollado, por el cumplimiento de los plazos o fechas determinadas de las tareas encomendadas y por la falta de apoyo de los profesores.

La siguiente tabla nos muestra los factores conformados con sus respectivos ítems (Tabla $\mathrm{N}^{\circ} 04$ ).

Tabla No 04

Factores de estrés e ítems asignados

\begin{tabular}{|l|c|c|}
\hline \multicolumn{1}{|c|}{ Factores } & Items & $\mathbf{N}^{\circ}$ de ítems \\
\hline 1. Baja estima académica & $25,29,34,36,40,41,42,43$ y 45 & 9 \\
\hline 2. Inseguridad al intervenir en público & $1,2,3,4$ y 9 & 5 \\
\hline 3. Deficiencia metodológica del profesorado. & $10,11,12,13,14,15,16,17$ y 21 & 9 \\
\hline 4. Sobrecarga del estudiante & $26,28,30,32,33,35,37,38$ y 39 & 9 \\
\hline 5. Malas relaciones sociales en el contexto académico & $44,48,49,51,52$ y 53 & 6 \\
\hline 6. Temor a los exámenes & $5,6,7$ y 8 & 4 \\
\hline $\begin{array}{l}\text { 7. Imposibilidad de participar en las decisiones respecto } \\
\text { del propio trabajo académico }\end{array}$ & $18,19,20$ y 46 & 4 \\
\hline 8. Carencia de valor de los contenidos de aprendizaje y estudio & $22,23,24$ y 47 & 4 \\
\hline 9. Falta de control sobre el propio rendimiento académico & 27,31 y 50 & 3 \\
\hline
\end{tabular}

Fuente: Resultados de Encuesta. Elaboración propia. 
Tabla No 05:

Matriz de componentes rotadas

\begin{tabular}{|c|c|c|c|c|c|c|c|c|c|c|}
\hline \multirow{2}{*}{\multicolumn{2}{|c|}{ Ítem }} & \multicolumn{9}{|c|}{ Componente } \\
\hline & & \multirow{2}{*}{$\begin{array}{c}1 \\
, 54\end{array}$} & \multirow[t]{2}{*}{2} & \multirow[t]{2}{*}{3} & \multirow[t]{2}{*}{4} & \multirow{2}{*}{5} & \multirow[t]{2}{*}{6} & \multirow[t]{2}{*}{7} & \multirow[t]{2}{*}{8} & \multirow[t]{2}{*}{9} \\
\hline P25 & No saber si mi ritmo de aprendizaje es el adecuado & & & & & & & & & \\
\hline P29 & Porque rindo claramente por debajo de mis conocimientos & 67 & & & & & & & & \\
\hline P34 & Porque no creo que pueda hacer frente a las exigencias de la carrera que estudio & ,57 & & & & & & & & \\
\hline P36 & Porque no creo que pueda lograr los objetivos propuestos & ,52 & & & & & & & & \\
\hline $\mathrm{P} 40$ & Porque desconozco si mi progreso académico es adecuado & ,63 & & & & & & & & \\
\hline P41 & Porque no sé cómo hacer bien las cosas & 63 & & & & & & & & \\
\hline P42 & Porque no sé qué hacer para que se reconozca mi esfuerzo y mi trabajo & ,66 & & & & ,41 & & & & \\
\hline P43 & Porque no tengo claro cómo conseguir que se valore mi dominio de las materias & ,62 & & & & ,42 & & & & \\
\hline P45 & Porque no sé que hacer para que se reconozca mi valía personal &, 54 & & & & ,49 & & & & \\
\hline P1 & Cuando me preguntan en clase & & ,79 & & & & & & & \\
\hline $\mathrm{P} 2$ & Si tengo que hablar en voz alta en clase & & ,80 & & & & & & & \\
\hline P3 & Al salir a la pizarra & & 80 & & & & & & & \\
\hline $\mathrm{P} 4$ & Al hacer una exposición o al hablar en público durante un cierto tiempo & &, 77 & & & & & & & \\
\hline P9 & Si tengo que exponer en público una opinión & & ,75 & & & & & & & \\
\hline P10 & $\begin{array}{l}\text { Cuando el profesor da la clase de una manera determinada y luego nos examina de } \\
\text { un modo poco coherente con esa forma de dar la clase }\end{array}$ & & &, 52 & & & & & & \\
\hline P11 & $\begin{array}{l}\text { Cuando los profesores no se ponen de acuerdo entre ellos (manifiestan claras dis- } \\
\text { crepancias entre ellos en temas académicos) }\end{array}$ & & &, 72 & & & & & & \\
\hline P12 & Cuando no me queda claro cómo he de estudiar una materia & & & 62 & & & & & & \\
\hline P13 & Cuando no tengo claro qué exigen en las distintas materias & & & ,68 & & & & & & \\
\hline P14 & $\begin{array}{l}\text { Cuando los profesores plantean trabajos, actividades o tareas que no tienen mucho } \\
\text { que ver entre sí (que son incongruentes) }\end{array}$ & & &, 59 & & & & & & \\
\hline P15 & Cuando el profesor no plantea de forma clara qué es lo que tenemos que hacer & & & 69 & & & & & & \\
\hline P16 & $\begin{array}{l}\text { Cuando el profesor plantea trabajos, actividades o tareas que son contradictorias } \\
\text { entre sí. }\end{array}$ & & & ,41 & & & & & & \\
\hline P17 & Cuando los distintos profesores esperan de nosotros cosas diferentes & & & ,45 & & & & & & \\
\hline $\mathrm{P} 21$ & $\begin{array}{l}\text { Cuando existe una clara falta de coherencia entre los contenidos de los distintos } \\
\text { materiales }\end{array}$ & & & ,40 & & & & ,60 & & \\
\hline P26 & Por el excesivo número de asignaturas que integran el plan de estudios de mi carrera &, 51 & & & & & & & & \\
\hline $\mathrm{P} 28$ & Por las demandas excesivas y variadas que se me hacen & ,49 & & & ,41 & & & & & \\
\hline P30 & $\begin{array}{l}\text { Por el escaso tiempo de que dispongo para estudiar adecuadamente las distintas } \\
\text { materias }\end{array}$ & & & &, 59 & & & & & \\
\hline P32 & $\begin{array}{l}\text { Por la excesiva cantidad de información que se me proporciona en clase, sin que se } \\
\text { indique claramente lo fundamental }\end{array}$ & & & & ,44 & & & & & \\
\hline P33 & $\begin{array}{l}\text { Por el excesivo tiempo que debo dedicarle a la realización de las actividades aca- } \\
\text { démicas }\end{array}$ & & & & ,53 & & & & & \\
\hline P35 & Porque no dispongo de tiempo para dedicarme a las materias todo lo necesario & & & & & & & ,39 & & \\
\hline P37 & Por la excesiva carga de trabajo que debo atender & & & & ,70 & & & & & \\
\hline P38 & Por el excesivo número de horas de clase diarias que tengo & & & & ,65 & & & & & \\
\hline P39 & Por el ritmo de trabajo o estudio que se nos exige & & & & ,75 & & & & & \\
\hline P44 & $\begin{array}{l}\text { Porque no tengo posibilidad alguna o muy escasa de dar mi opinión sobre la meto- } \\
\text { dología de enseñanza de las materias del plan de estudios }\end{array}$ & & & & & ,50 & & & ,42 & \\
\hline P48 & Por los conflictos en las relaciones con otras personas (profesores, compañeros...) & & & & & ,60 & & & & \\
\hline P49 & Por la excesiva competitividad existente en clase & & & & & ,43 & & & & \\
\hline P51 & Por la falta de apoyo de los compañeros & & & & & 60 & & & & \\
\hline
\end{tabular}




\begin{tabular}{|c|c|c|c|c|c|c|c|c|c|c|}
\hline \multirow{2}{*}{\multicolumn{2}{|c|}{ Ítem }} & \multicolumn{9}{|c|}{ Componente } \\
\hline & & \multirow[t]{2}{*}{1} & \multirow[t]{2}{*}{2} & \multirow[t]{2}{*}{3} & \multirow[t]{2}{*}{4} & \multirow{2}{*}{\begin{tabular}{|l|}
5 \\
, 61
\end{tabular}} & \multirow[t]{2}{*}{6} & \multirow[t]{2}{*}{7} & \multirow[t]{2}{*}{8} & \multirow[t]{2}{*}{9} \\
\hline P52 & Por la ausencia de un buen ambiente en clase & & & & & & & & & \\
\hline P53 & Por la existencia de favoritismos en clase & & & & & ,58 & & & & \\
\hline P5 & Al hablar de los exámenes & & & & & & ,46 & & & \\
\hline P6 & Cuando tengo exámenes & & & & & & ,78 & & & \\
\hline $\mathrm{P} 7$ & Mientras resuelvo los exámenes & & & & & & ,78 & & & \\
\hline P8 & Cuando se acercan las fechas de los exámenes & & & & & & ,70 & & & \\
\hline $\mathrm{P} 18$ & Cuando el profesor espera de nosotros que sepamos cosas que no nos ha enseñado & & & & & & & ,61 & & \\
\hline P19 & $\begin{array}{l}\text { Cuando el profesor da por hecho que tenemos conocimientos que en realidad no } \\
\text { tenemos }\end{array}$ & & & & & & & ,63 & & \\
\hline $\mathrm{P} 20$ & $\begin{array}{l}\text { Cuando el profesor plantea exámenes claramente incongruentes con lo estudiado/ } \\
\text { enseñado }\end{array}$ & & & & & & & ,65 & & \\
\hline P46 & $\begin{array}{l}\text { Porque las posibilidades de opinar sobre el procedimiento de evaluación de las asig- } \\
\text { naturas del plan de estudios son muy escasas o nulas }\end{array}$ & & & & & ,50 & & & & \\
\hline $\mathrm{P} 22$ & Que las asignaturas que cursamos tienen escaso interés & & & & & & & & ,71 & \\
\hline $\mathrm{P} 23$ & Que lo que estoy estudiando tiene una escasa utilidad futura & & & & & & & & ,66 & \\
\hline $\mathrm{P} 24$ & Que las clases a las que asisto son poco prácticas & & & & & & & &, 53 & \\
\hline P47 & $\begin{array}{l}\text { Porque no está en mi mano plantear los trabajos, tareas o actividades como me gus- } \\
\text { taría }\end{array}$ & & & & & & & & ,41 & \\
\hline P27 & $\begin{array}{l}\text { Porque los resultados obtenidos en los exámenes no reflejan, en absoluto, mi traba- } \\
\text { jo anterior de preparación ni el esfuerzo desarrollado }\end{array}$ & ,44 & & & & & & & & \\
\hline P31 & $\begin{array}{l}\text { Por el cumplimiento de los plazos o fechas determinadas de las tareas encomen- } \\
\text { dadas }\end{array}$ & & & & & & & & & ,91 \\
\hline \multirow[t]{3}{*}{ P50 } & Por la falta de apoyo de los profesores & & & & & & & & & 87 \\
\hline & \multicolumn{10}{|c|}{$\begin{array}{l}\text { Ítems con menos del 50\% de variabilidad explicada en el factor asignado, se puede reasignar a otro factor, (se le esta asignando al factor } \\
\text { sombreado) }\end{array}$} \\
\hline & $\begin{array}{l}\text { Son ítems que representan a } 2 \text { factores, se deja en el factor que guarde mejor relacic } \\
\text { factor) }\end{array}$ & & & & & & & & & \\
\hline
\end{tabular}

Fuente: Resultados de Encuesta. Elaboración propia. 


\section{Para la determinación del Nivel de Estrés:}

Para ver el grado de estrés que presentan los estudiantes se sumaron las puntuaciones marcadas en cada ítem, obteniéndose una calificación final por cada estudiante, luego se clasifico en 3 niveles: las que presentan bajo grado de estrés, medio grado de estrés $\mathrm{y}$ alto grado de estrés (Ver Tabla $\mathrm{N}^{\circ} 06$ ). También se consideraron puntuaciones por cada factor y se clasificaron en los 3 niveles de estrés.

Finalmente para ver cuáles son los factores estresores que más influyen en el desarrollo de la carrera de los estudiantes se calcularon los resultados en base a cada factor.

Tabla No 06

Rangos de estimación de niveles de estrés

\begin{tabular}{|l|c|c|c|}
\hline \multirow{2}{*}{ Factores } & \multicolumn{3}{c|}{ Nivel de Estrés } \\
\cline { 2 - 4 } & Bajo & Medio & Alto \\
\hline Baja estima académica & {$[09-20]$} & {$[21-32]$} & {$[33-45]$} \\
\hline Intervenciones en público & {$[05-11]$} & {$[12-17]$} & {$[18-25]$} \\
\hline Deficiencia metodológica del profesorado. & {$[09-20]$} & {$[21-32]$} & {$[33-45]$} \\
\hline Sobrecarga del estudiante & {$[09-20]$} & {$[21-32]$} & {$[33-45]$} \\
\hline Malas relaciones sociales en el contexto académico & {$[06-13]$} & {$[14-21]$} & {$[22-30]$} \\
\hline Temor a los exámenes & {$[04-08]$} & {$[09-14]$} & {$[15-20]$} \\
\hline $\begin{array}{l}\text { Imposibilidad de participar en las decisiones respecto del } \\
\text { propio trabajo académico }\end{array}$ & {$[04-08]$} & {$[09-14]$} & {$[15-20]$} \\
\hline Carencia de valor de los contenidos de aprendizaje y estudio & {$[04-08]$} & {$[09-14]$} & {$[15-20]$} \\
\hline Falta de control sobre el propio rendimiento académico & {$[03-06]$} & {$[07-10]$} & {$[11-15]$} \\
\hline Total general & {$[53-123]$} & {$[124-193]$} & {$[194-265]$} \\
\hline
\end{tabular}

Fuente: Resultados de Encuesta. Elaboración propia.

\section{RESULTADOS}

- Con respecto al total de las dimensiones presentadas como resultado del análisis: Los estudiantes señalaron como las más recurrentes sólo a la mitad de ellas (Ver Tabla $\mathrm{N}^{\circ} 07$ ). De esta mitad, el $44 \%$ son las más recurrentes, en más del 50\% del alumnado encuestado, presentándose éstas en un grado medio o alto, siendo éstas, en orden porcentual descendente : "la "imposibilidad de participar en las decisiones del propio trabajo, la "Falta de control sobre el propio rendimiento académico", la "sobrecarga del estudiante" y la "deficiencia metodológica del profesorado”. En contra parte, las dos menos recurrentes y percibidas como las menos estresantes por todo el alumnado encuestado es "malas relaciones sociales en el contexto académico y la baja estima académica.
- Con respecto a los niveles de estrés resultantes, de acuerdo a la escala establecida: el estrés se presenta en el nivel bajo en un 50.7\%, en el nivel medio en un $46.5 \%$ y en el nivel alto en un $2.8 \%$ del alumnado encuestado.

- Con respecto a la presencia del estrés en la totalidad de estudiantes encuestados, en lo general, está marcadamente dividido, pues la mitad de los estudiantes encuestados (49.3\%), refirieron que el estrés, está presente en ellos y los afecta en un nivel medio (46.5\%) oun nivel alto o crítico (2.8\%).

- Con respecto al grado o nivel crítico de afectación del estrés. Se concluye que la mayoría de estudiantes $(34.6 \%)$ se siente afectado en un alto grado de estrés cuando debe realizar sus "intervenciones en público", seguido de aquellos que señalaron 
Tabla No 07

Niveles Estresores

\begin{tabular}{|c|c|c|c|c|}
\hline \multirow{2}{*}{ Factores } & \multicolumn{3}{|c|}{ Nivel de Estrés } & \multirow{2}{*}{ Total } \\
\hline & Bajo & Medio & Alto & \\
\hline \multirow{2}{*}{ Baja estima académica } & 187 & 134 & 32 & 353 \\
\hline & $53.0 \%$ & $38.0 \%$ & $9.1 \%$ & $100.0 \%$ \\
\hline \multirow{2}{*}{ Intervenciones en público } & 183 & 122 & 48 & 353 \\
\hline & $51.8 \%$ & $34.6 \%$ & $13.6 \%$ & $100.0 \%$ \\
\hline \multirow{2}{*}{ Deficiencia metodológica del profesorado. } & 173 & 154 & 26 & 353 \\
\hline & $49.0 \%$ & $43.6 \%$ & $7.4 \%$ & $100.0 \%$ \\
\hline \multirow{2}{*}{ Sobrecarga del estudiante } & 156 & 171 & 17 & 353 \\
\hline & $44.2 \%$ & $48.4 \%$ & $7.4 \%$ & $100.0 \%$ \\
\hline \multirow{2}{*}{ Malas relaciones sociales en el contexto académico } & 212 & 124 & 18 & 353 \\
\hline & $61.1 \%$ & $35.1 \%$ & $4.8 \%$ & $100.0 \%$ \\
\hline \multirow{2}{*}{ Temor a los exámenes } & 186 & 149 & 53 & 353 \\
\hline & $52.7 \%$ & $42.2 \%$ & $5.1 \%$ & $100.0 \%$ \\
\hline \multirow{2}{*}{$\begin{array}{l}\text { Imposibilidad de participar en las decisiones } \\
\text { respecto del propio trabajo académico }\end{array}$} & 84 & 216 & 20 & 353 \\
\hline & $23.8 \%$ & $61.2 \%$ & $15.0 \%$ & $100.0 \%$ \\
\hline \multirow{2}{*}{$\begin{array}{l}\text { Carencia de valor de los contenidos de aprendizaje } \\
\text { y estudio }\end{array}$} & 180 & 153 & 20 & 353 \\
\hline & $51.0 \%$ & $43.3 \%$ & $5.7 \%$ & $100.0 \%$ \\
\hline \multirow{2}{*}{$\begin{array}{l}\text { Falta de control sobre el propio rendimiento } \\
\text { académico }\end{array}$} & 119 & 196 & 38 & 353 \\
\hline & $33.7 \%$ & $55.5 \%$ & $10.8 \%$ & $100.0 \%$ \\
\hline \multirow{2}{*}{ Total general } & 179 & 164 & 10 & 353 \\
\hline & $50.7 \%$ & $46.5 \%$ & $2.8 \%$ & $100.0 \%$ \\
\hline
\end{tabular}

Fuente: Resultados de Cuestionario. Elaboración propia.

que más bien es la "imposibilidad de participar en las decisiones respecto de su propio trabajo" lo que les causa un mayor nivel de estrés (15\%). Otras situaciones en las que el estrés produce un malestar emocional agudo en los estudiantes es la "falta de control sobre el propio rendimiento académico", manifestado en el $10 \%$ del alumnado, así como la sobrecarga de labores académicas que debe afrontar el estudiante en su formación, que afecta a un importante $7.4 \%$ de los estudiantes.

- En lo que respecta a resultados de afectación del estrés por Escuelas Académico Profesionales (EAP): Se concluye que la EAP que concentra mayor nivel de estrés, en sus estudiantes, en comparación con las otras dos EAP, es la EAP de Auditoría Empresarial y del Sector Público, en un $60 \%$, de los cuales, el $54.3 \%$ lo percibe un nivel de afectación medio y el $2.6 \%$ lo percibe en un nivel alto.
- En cuanto a los niveles de estrés por Semestre académico. Los estudiantes del noveno semestre académico quienes a su vez, representan el $39.4 \%$ del total de la muestra encuestada, son los que experimentan en mayor número el estrés, siendo su grado de afectación nivel medio en un $51.1 \%$ $y$ en un nivel alto un $1.4 \%$.

- En los resultados comparativos, por sexo, se obtuvo que las mujeres, que en comparación con los varones, representan el 54\% de la población encuestada, son las que mayoritariamente, experimentan estrés, siendo de grado medio en un $48.7 \%$ y en grado alto, un $3.7 \%$.

- En los resultados comparativos por edad, los estudiantes de entre 22 y 24 años, que de acuerdo al total de la muestra encuestada, representan el $32.2 \%$, son los que mayoritariamente presentan la afectación del estrés, el cual es percibido en un nivel medio en un $49.1 \%$ y en un nivel alto, un $3.5 \%$. 
- En los resultados comparativos por condición laboral, los estudiantes que trabajan, que representan el $37.4 \%$ de total de la muestra, son los que mayoritariamente están afectados por el estrés, presentándose en ellos el estrés en un nivel medio para un $52.3 \%$ y en un nivel alto para un $1.5 \%$

\section{CONCLUSIONES:}

1. De acuerdo con los resultados expuestos, es posible afirmar que las principales situaciones percibidas como las más estresantes por los estudiantes de la Facultad de Ciencias Contables, se relacionan sobre todo con los temas de falta de control sobre algunos aspectos del sistema formativo impuestos, como la evaluación, la sobrecarga académica y las deficiencias metodológicas que presentan los docentes, que según su percepción, ello les genera tensión e incapacidad pues definitivamente el estilo de impartir la enseñanza marca las pautas de interacción con sus docentes yles define claramente una línea de desempeño a ejercer.

2. El Semestre Académico que cursa el estudiante también influye en el nivel de presencia del estrés, siendo éste, según los resultados observados, mayor al final de los estudios de la Carrera. En el caso del estudio realizado, el primero y último semestre, son períodos de transición y de cambio en sus propias realidades, en la adolescencia y la adultez joven.

3. Si la mitad de la población estudiantil presenta el síndrome del estrés, es necesario que la institución educativa aplique programas o estrategias de manejo del estrés sobre todo en la población que lo presenta en magnitud crítica, como una de los mecanismos de apoyo al estudiante, en su rendimiento, durante la carrera, ayudándolos a enfrentarlo sobre todo en los alumnos del primer Semestre, por estar ellos iniciando un proceso de formación profesional y de adaptación, que constituye parte de su plan de vida y a los del último semestre, por estar involucrados en el desarrollo de sus prácticas preprofesionales, parte indispensable de la formación integral profesional de todo futuro contador.
4. Si bien los resultados presentados, reflejan una parte de la realidad del estrés de los estudiantes de la Facultad de Ciencias Contables, es importante su profundización y contrastación con otras variables del rendimiento académico, a efectos de verter juicios de valor más de fondo en torno al estrés académico.

\section{REFERENCIAS BIBLIOGRÁFICAS:}

1. BARRAZA M., A. (2006). Un modelo conceptual para el estudio del estrés académico. Revista. Electrónica de Psicología Iztacala. Vol 9 (3).

2. CABANACH, R.G., VALLE A., PIÑERO, I., RODRÍGUEZ, S. y GARCÍA, M. (2009). Diseño y validación de un cuestionario de gestión motivacional. Revista de Psicodidáctiva . Vol. 14 (1), p. 29-47.

3. CABANYES T.,J. (2012). La Salud Mental en el mundo de hoy. Málaga, España: Editorial EUNSA.

4. DÍAZ M., Y. (2010). Estrés académico y afrontamiento en estudiantes de Medicina. Humanidades Médicas. Vol. 10 (1).

5. FELDMAN, L.et al. (2008). Relaciones entre estrés académico, apoyo social, salud mental y rendimiento académico en estudiantes universitarios venezolanos. Grupo Psicología y Salud. Vol. 7 (3) p. 739-751.

6. HERRANZ-B., J., REIG F. A., CABRERO, G., J. (2006). La prevalencia del estrés laboral asistencial entre los profesores universitarios. Análisis y Modificación de Conducta, Vol. 32, p. 746-748.

7. MARTÍN M., I. (2007). Estrés académico en estudiantes universitarios. Apunte de Psicología Vol. 25 (1), p.87-99.

8. MARTíN G., M. \& TRUjILlO A., F. (2012). Evaluación del estrés académico percibido por estudiantes de grado de ingenierías industriales en la universidad de Málaga. Innovagogía, p.1518.

9. RODRÍGUEZ G., M., SAN MIGUEL S., M., MUÑOZ M., A., RODRÍGUEZ R., C. (2014). El estrés en estudiantes de Medicina al inicio y al final de su formación académica. Revista Iberoamericana de Educación. No 66. P.105-122. 